\title{
Verbal indices of conceptual complexity: Manipulation by instructions
}

\author{
PETER SUEDFELD, ${ }^{1}$ Rutgers - The State University, New \\ Brunswick, N.J. 08903
}

A critique of one prearranged 'response" changed Ss' scores on two measures of conceptual complexity. The modifiability of these scores, not tested directly before, has implications for researchers in the area.

Ever since Schroder and his co-workers (Harvey, Hunt, \& Schroder, 1961; Schroder, Driver, \& Streufert, 1967) have described the conceptual structures which underlie individual tendencies in information processing, the question of structural modification has been of some interest. It has been assumed that childhood environment determines whether the cognitive system becomes simple (rigid, dichotomized, insensitive to data) or complex (flexible, combinatorial, information-oriented), and environmental parameters such as information load have been shown to interact with structural disposition to determine behavioral complexity (see Schroder et al, 1967). Until recently, however, "no studies have... directly and objectively induced differential degrees of conceptual level" (Lee, 1968, p. 45); and even the few short-range training procedures (Schroder, in press) have not used the standard conceptual complexity measures as dependent variables. This was done in the present study, brief evaluations of a sample response being varied to elicit changes in the complexity scores of other Ss in the group.

\section{METHOD}

The two standard instruments for measuring conceptual complexity are the Paragraph Completion Test (PCT) and the Impression Formation Test (IFT). The former is scored on the basis of completions of items such as "Parents..." and "When I am criticized ... "; the latter, in terms of S's ability to integrate two somewhat incomparable adjective lists into the description of a stimulus person (details are given in Schroder et al, 1967).

A group of 132 undergraduates was split at the median score on one of equivalent halves of the PCT, and 22 complex and 22 simple responders were assigned randomly to each of three conditions. The experimental treatment, several weeks after original testing, consisted of group administration of one of the two IFT items. After all Ss had finished answering, one volunteer (actually a confederate) was asked to read her response "to give everybody an idea of how to do the task." One of three instructions was given to the medium-complexity response read aloud:

Neutral Instruction (NI): "Well, that's not too bad-we get a fair number of responses that are somewhat similar."

Complex Instructions ( $\mathrm{Cl}$ ): "Well, that's not too bad-we get a fair number of answers that are somewhat similar. The major fault of your answer was that you've oversimplified the situation by not adequately considering all of: the important factors. You have to take all of the important things together into account; that's the only way to go to the heart of the matter. When writing your answers, remember that these people are complex human beings; try not to ignore or underemphasize the different items of important information, and try to avoid winding up with simple and categorical judgments."
Simple Instructions (SI): "Well, that's not too bad-we get a fair number of answers that are somewhat similar. The major fault of your answer was that you've overcomplicated the situation by trying to take into account too many unimportant factors. You have to decide what the really important things are; that's the only way to go to the heart of the matter. When writing your answers, remember that these people can be described quite simply and easily; try to avoid getting lost in all the information which is really pretty unimportant and then winding up with vague and complicated judgments."

Then all Ss did the remaining IFT item and the second half of the PCT.

\section{RESULTS AND DISCUSSION}

The NI group showed no significant change in complexity from the pre- to the post-instruction measures. For the other two groups, IFT change showed a main effect for conceptual complexity $[F(1,126)=4.49, p<.05]$; complex $\mathrm{Cl}$ and $\mathrm{SI} S \mathrm{Ss}$ became even morc complex, while simple Ss became much simpler after $\mathrm{SI}$ and slightly less so after CI. Original complexity also gave a significant effect on the PCT $[F(1,126)=53.98, p<.001)$ simple $S s$ becoming more complex and complex Ss becoming simpler. The treatments effect was also significant $[F(1,126)=3.94, p<.05]$, SI resulting in smaller complexity increments for simple $\mathrm{Ss}$ and greater changes toward simplicity for complex Ss than did $\mathrm{Cl}$.

The increased complexity of complex Ss on the IFT, regardless of instructions, may have been the effect of salience on the Ss' original response tendencies. Simple Ss, on the other hand, responded to instructions. This result was unexpected, since complex Ss theoretically should be more able to function simply than simple Ss to function in complex ways. It has been established, however, that simple Ss rely more on external determinants to guide behavior (Schroder et al, 1967), which may have mediated the change. Some of the PCT change was the result of regression toward the mean. Nevertheless, IFT instructions did generalize to PCF responses, showing that complexity scores can be changed even by a very brief and low-key experimental procedure. Verbal indices of conceptual complexity seem to be more malleable than has been realized until now. This finding emphasizes the importance of standardized test instructions; the effects of various kinds of instructional set, and the generality and duration of the changes, should be investigated further.

\section{REFERENCES}

HARVEY, O. J., HUNT, D. E., \& SCHRODER, H. M. Conceptual systems and personality organization. New York: Wiley, 1961.

LFE, R. E., III. Dispositional and induced information processing structures. Unpublished Ph.D. dissertation, Princeton University, 1968.

SCHRODER, H. M. Conceptual complexity and personality organization. In H. M. Schroder and P. Suedfeld (Eds.), Personality: Information processing modcls. New York: Ronald, in press.

SCHRODER, H. M.. DRIVER, M. J., \& STRFUFERT, S. Human information processing. New York: Holt, Rinehart, and Winston. 1967.

NOTE

1. This research was supported by internal funds from the University of Illinois and Rutgers - The State University. The assistance of Richard L. Hagen is gratefully acknowhedged. 